\title{
Development of a spectrometer for airborne measurement of droplet sizes in clouds
}

E. Porcheron

emmanuel.porcheron@irsn.fr

P. Lemaitre

J. Van Beeck

R. Vetrano

M. Brunel

G. Grehan

L. Guiraud
Institut de Radioprotection et de Sûreté Nucléaire (IRSN), BP 68, 91192 Gif-sur-Yvette, France

Institut de Radioprotection et de Sûreté Nucléaire (IRSN), BP 68, 91192 Gif-sur-Yvette, France

Institut von Karman, B-1640 Rhode-Saint-Genese, Belgium

Institut von Karman, B-1640 Rhode-Saint-Genese, Belgium

Normandie Université, UMR 6614 CORIA, CNRS, Université et INSA de Rouen, Av. de 1'Université, 76801 Saint-Etienne du Rouvray cedex, France

Normandie Université, UMR 6614 CORIA, CNRS, Université et INSA de Rouen, Av. de l'Université, 76801 Saint-Etienne du Rouvray cedex, France

Service des Avions Francais Instrumentés pour la Recherche en Environnement, BP 20034, 31270 Cugnaux, France

The objective of this article is to present the development and the validation in flight of an airborne probe that can measure in clouds the size of droplets whose diameters are in the range $[20 \mu \mathrm{m} ; 200 \mu \mathrm{m}]$.

[DOI: http://dx.doi.org/10.2971/jeos.2015.15030]

Keywords: Interferometric laser imaging laser for droplets sizing, two-phase flows, clouds, airborne instrumentation

\section{INTRODUCTION, OBJECTIVE AND MOTIVATION}

In situ characterization of precipitation onset with airborne instruments is one of the key challenges in cloud microphysics. Droplets growing by condensation of water vapour reach a critical diameter slightly larger than $20 \mu \mathrm{m}$, above which they can collide and coalesce into precipitation nuclei. The main obstacle to detection of precipitation embryos is their relatively small concentration (a few embryos per $\mathrm{dm}^{3}$ ) compared to that of droplets (several hundreds of thousands per $\mathrm{dm}^{3}$ ). The Forward Scattering Spectrometer Probe (FSSP) size range ends at 47 or $95 \mu \mathrm{m}$, depending on the version. Above $40 \mu \mathrm{m}$, its sampling section is too small to perform statistically significant measurements of big droplet concentrations [1, 2]. Generally, the probes for Particle Measurement Systems have a coarse resolution for particles below $100 \mu \mathrm{m}$, and a poorly defined sampling section for particles in the range of size $100 \mu \mathrm{m}$ to $200 \mu \mathrm{m}$. The Cloud Particle Imager (CPI) has a better resolution, but the measurements are not continuous and there is no automatic processing of the images [3]. The Phase Doppler Particle Analyzer (PDPA, or Phase Doppler Interferometer PDI) provides absolute measurements of droplet sizes [4], but the airborne version has not yet demonstrated the expected performance in terms of measuring droplet sizes above $100 \mu \mathrm{m}$ [5]. The sampling section of PDI, which corresponds to the intersection of the beams, is relatively small $\left(<1 \mathrm{~mm}^{2}\right)$. A difficulty of this system is that the beams must be very accurately aligned (which is difficult in the harsh environment of airborne operations). Airborne droplet spectrometers, for diameters smaller than $40 \mu \mathrm{m}$, rely on light scattering. In order to avoid droplet coincidences, their sampling section is narrow $\left(0.5 \mathrm{~mm}^{2}\right)$, thus limiting the detection of diluted particles as precipitation nuclei. Above $40 \mu \mathrm{m}$ in diameter, droplet spectrometers rely on particle shadowing. Both scattering and shadowing instruments are sensitive to attenuation of the incident laser light and require frequent calibrations.

The objective of this article is to present the development of an airborne probe undertaken within the ALIDS (Airborne Laser Interferometric Drop Sizer) project. It is done within the context of the EUFAR network (EUropean Facility for Airborne Research) funded by the 7th European Framework Program (2007-2013), and coordinated by Météo-France [6]. The design parameters of the spectrometer are specified in order to be able to characterize droplet diameters in clouds from $20 \mu \mathrm{m}$ to $200 \mu \mathrm{m}$ with a large probe volume. The steps of the development of the prototype instrument are presented and explained. They include: design and optimization of the optical setup, probe development, integration of probe components, probe qualification in laboratory and demonstration of airborne measurement during a flight test. The principle applied in this project for drop sizing is currently referred to as Interferometric Laser Imaging for Droplet Sizing (ILIDS). It is detailed in Figure 1. This technique offers a relatively large sam- 


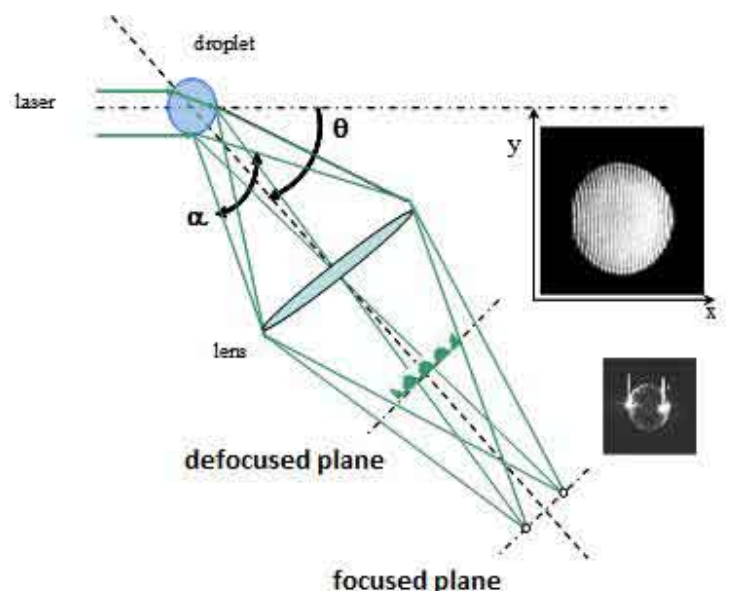

FIG. 1 Typical Interferometric Laser Imaging for Droplet Sizing (ILIDS) set-up. The two additional images are the in-focus image of a droplet where two bright spots are predominant, and an out-of-focus image of the same droplet where interference fringes are observed.

pling volume. It provides absolute measurements of drop size, using interference fringes detection whose spatial frequency is linked to droplet diameter with a linear and robust relation. In our case, the thickness of the laser sheet is equal to $0.1 \mathrm{~cm}$. The sampling section is $100 \mathrm{~cm}^{2}(10 \mathrm{~cm} \times 10 \mathrm{~cm})$. The sample volume is thus $10 \mathrm{~cm}^{3}$. ILIDS technique appears particularly well adapted to a diluted environment, such as clouds, for droplet sizes larger than $20 \mu \mathrm{m}$. The cloud volume sampled by ILIDS is then dependent on the repetition rate of the laser which can easily reach from $30 \mathrm{~Hz}$ for a very compact air-cooled Nd:YAG laser up to more than $100 \mathrm{~Hz}$ for a water-cooled laser or a Diode-Pumped Solid State laser. Considering an acquisition frequency of $30 \mathrm{~Hz}$ with a sampling volume equal to $10 \mathrm{~cm}^{3}$, we test a volume of $300 \mathrm{~cm}^{3}$ every 100 meters (for an aircraft flying at $100 \mathrm{~m} / \mathrm{s}$ ).

\section{GENERAL BACKGROUND OF THE ILIDS TECHNIQUE}

Interferometric Laser Imaging for Droplet Sizing is a technique that provides the absolute instantaneous size and spatial distributions of transparent spherical particles (droplets and bubbles) in a section of a flow. It was first introduced by König et al [7] and further improved by Glover et al [8] for image acquisition and data processing. Figure 1 shows the typical experimental setup.

A laser sheet is sent toward a group of droplets. Light scattered by the droplets is collected by a receiving optic. Different configurations can be found in the literature: the scattering angle ordinarily chosen is $\theta=66^{\circ}[8,9]$; but experiments can be performed and interpreted as well with $\theta=90^{\circ}$ [10]. The glare points associated with reflected and refracted rays can be observed in the plane where their images are in-focus (see in Figure 1 the in-focus image of the droplet). The size of the particle can be determined by measuring the distance between these two points [11]. This method requires a very high resolution to determine with a good accuracy droplet size. Another way is to observe the image of the droplets in a defocused plane.
Therefore, for ILIDS diagnostics, images are captured by a CCD camera positioned in a defocused plane (out-of-focus imaging). The camera observes the interferometric pattern of the light scattered by the particles. Each droplet is associated with a fringe pattern in a circle. Using a geometrical optics approach, König et al [7] calculated the optical path difference between reflected and refracted rays. They deduced the relation between inter-fringe spacing, droplet diameter and index of refraction (Eq. (1)). For each droplet, the droplet diameter $(d)$ is linked to the number of fringes $(N)$ by a factor which depends on aperture angle of the collecting system $(\alpha)(\alpha$ is linked to the receiving optics parameters), scattering angle $(\theta)$, refractive index of the droplet $(m)$, and wavelength of the laser sheet $(\lambda)$ according to :

$$
d=\frac{2 \lambda N}{\alpha}\left(\cos (\theta / 2)+\frac{m \sin (\theta / 2)}{\sqrt{m^{2}+1-2 m \cos (\theta / 2)}}\right)^{-1}
$$

Up to now, ILIDS technique was commonly used in laboratories for fluid mechanics research, such as internal combustion or rocket engines, spray and nuclear safety $[12,13]$. Progress in optics technology, such as miniaturization of high-energy Qswitched lasers and CCD sensors, allows the ILIDS technique to be transferred to aircraft for performing airborne measurements. ILIDS optical setup is simple, similar to PIV (Particles Image Velocimetry) technique. Its adjustment is quite easy due to the fact that most of the setup parameters are mechanically fixed. Coherent light which is needed for this technique is generated by a frequency-doubled, Nd:YAG pulsed laser $(\lambda=532 \mathrm{~nm})$ with high repetition rate. It is polarized perpendicularly to the scattering plane in order to increase fringe visibility. The laser beam is extended using a cylindrical lens to create a laser sheet. The laser pulse duration is less than 10ns. It means that ILIDS technique is able to measure an instantaneous droplet size field without influence of aircraft velocity through the cloud. In addition, ILIDS technique is not sensitive to the variation of droplets refractive index in the range of airborne conditions, i.e. for temperatures between $-40^{\circ} \mathrm{C}$ (super-cooled droplets) and $40^{\circ} \mathrm{C}$. Images are acquired by a high-speed CCD camera which is synchronized with the laser frequency. Besides, ILIDS technique offers the possibility to discriminate water droplets from ice aerosols for which speckle-like patterns appear.

\section{OPTICAL OPTIMIZATION OF ILIDS SETUP FOR AIRBORNE INTEGRATION AND CONSTRAINTS}

ILIDS technique is a well-known technique in laboratories for applications dealing with bubble, droplet or spray characterization. The application of this technique for airborne measurements using an external probe fixed on an aircraft imposes several design constraints. They must be taken into account from the beginning of the airborne instrument definition. The design drivers have to meet the following main requirements:

- large probe volume (at least $100 \mathrm{~cm}^{3}$ ) in order to increase the probability of detecting precipitation embryos,

- range of droplet size distributions from $20 \mu \mathrm{m}$ to $200 \mu \mathrm{m}$, 
- miniaturization of the ILIDS setup to reduce the airborne probe size and weight for reaching an acceptable drag coefficient of the instrument. This point is particularly important due to the difficulty and the cost of certifying new instruments on aircrafts,

- best signal-to-noise ratio in order to use the lowest laser power.

The following approach is then based on the complementary use of simulations and experiments performed on an optical table. If some parameters can be optimized using simulations, others, such as the required laser power, have to be determined by experiments. The integration constraints of a laser in an airborne instrument are the major ones due to the fact that sizing, mass and power supply available in an airborne probe are very limited. Even if laser capabilities are continuously increasing, the ILIDS optical setup has to be optimized in order to have the best efficiency: best out-of-focus image quality with lowest laser energy.

Although the scattering angle $90^{\circ}$ could offer easier integration of the optical assembly, the angle close to the Brewster angle offers better fringe contrast. At the angle of $66^{\circ}$, the intensities of the scattering orders corresponding to reflected $(p=0)$ and refracted $(p=1)$ light are equal, for an incident wave polarized perpendicularly to the scattering plane. A numerical code has been developed in order to simulate any kind of ILIDS setup. It is based on the use of generalized Huygens Fresnel integrals associated to transfer matrices. This simulator offers important perspectives in the design, realization, and calibration of ILIDS instruments, as optimization of airborne instrument, or in situ measurements in real conditions. Components as optical windows, cylindrical lenses, pipes, and other optical components can be taken into account [14]. Using the transfer-matrix based formalism that we developed, the diameter of the droplet can be linked to the fringe frequency and to the optical system through the general relation

$$
d=2 \lambda B_{t o t} F \quad\left(\cos (\theta / 2)+\frac{m \sin (\theta / 2)}{\sqrt{m^{2}+1-2 m \cos (\theta / 2)}}\right)^{-1}
$$

where $F$ is the fringe frequency and $B_{t o t}$ is the B parameter of the total transfer matrix between the droplets and the CCD sensor [14]. ILIDS image processing is one of the major challenges for airborne application because the instrument should give the droplet size distribution in real-time during the flight. Indeed, due to flight hour cost, it is crucial to be able to adapt the flight plan according to measurements already performed. In particular, acquisition rate should exceed $10 \mathrm{~Hz}$, with synchronization of laser shot and image acquisition. Keep in mind that the probe provides one measurement every $10 \mathrm{~m}$ traveled by the aircraft. The analysis of ILIDS images must be shorter than $0.1 \mathrm{~s}$. Different approaches exist for ILIDS image processing. The common one is to analyze each interferogram of the image by Fast 2D-Fourier Transform in order to determine the number of interference fringes. Using Eq. (1), the size of each droplet can then be determined. In addition, this approach allows giving the location and possibly the velocity of each droplet recorded on a couple of images. This kind of processing is quite long. In addition, for airborne application, the knowledge of all those parameters is not useful. Another approach, developed by Maeda [15], consists in analyzing a one-dimensional image obtained by optical compression made with a cylindrical lens. This approach is well adapted for dense two-phase flows but can have some limitations when interference fringes are not completely vertical. In our case, we will only determine global droplet size distribution over the whole image. We do not look for any local analysis per droplet but we need a global image analysis. In order to realize a high-speed analysis of all interferograms present on one image, an analysis based on a Fast Fourier Transform of the image has been developed [16]. The ILIDS optical setup developed in our laboratory and its global image processing have been validated by comparative measurements with PDPA (Phase Doppler Particle Analyzer). These quantitative comparisons have validated our algorithm, as was presented in reference [16].

\section{AIRBORNE PROBE DESIGN AND CONSTRUCTION}

The architecture of ILIDS setup must satisfy the parameters defined above. It must also be integrated in a small volume for having a minimum drag coefficient. It is necessary to limit the weight of the instrument to reduce the costs incurred by its certification on aircraft. The geometry of the sensor is symmetrical about the plane containing the velocity vector of the aircraft. Moreover, the measurement plane should contain the main component of the aircraft velocity vector. Even if the laser pulse duration, shorter than $10 \mathrm{~ns}$, limits the movement of the drop in the measurement volume to a few microns (aircraft speed range between 100 and $200 \mathrm{~m} / \mathrm{s}$ ), it is preferable that this displacement takes place collinearly to the laser sheet and not perpendicularly. In addition, the measurement volume must be at a distance larger than $10 \mathrm{~cm}$ from any surface of the probe, to be outside the boundary layer of the probe. The electronic equipment, including ILIDS assembly, laser head and camera, must be fully integrated in the sensor to limit their exposure to thermal stress specific to flight conditions at high altitude. A diagram showing the front view of the Airborne interferometric Laser Imaging for Droplet Sizing probe (ALIDS), and revealing its symmetric design, is presented in Figure 2(a). After mirror M4, the laser beam crosses the centerline, as does the optical axis of the camera before mirror M3. Thus, a quite symmetric layout can be achieved for $\theta=66^{\circ}$ when setting angle $\tau$ to $6^{\circ}$ for mirrors M1 and M2. The probe volume is thus centered on the probe's symmetry axis. The 3D design of the instrument presented in Figure 2(b) has been achieved by company COMAT (COncept Mecanique Assistance Technique) [17] according to all following designed drivers: geometry of optical setup for ILIDS; sizing of the different components including laser head, camera, lenses, mirrors; weight limitation for integration on the aircraft (ATR-42); drag effort induced by the probe during flight; mechanical justification including vibration analysis and mechanical resistance of the probe. 
a)

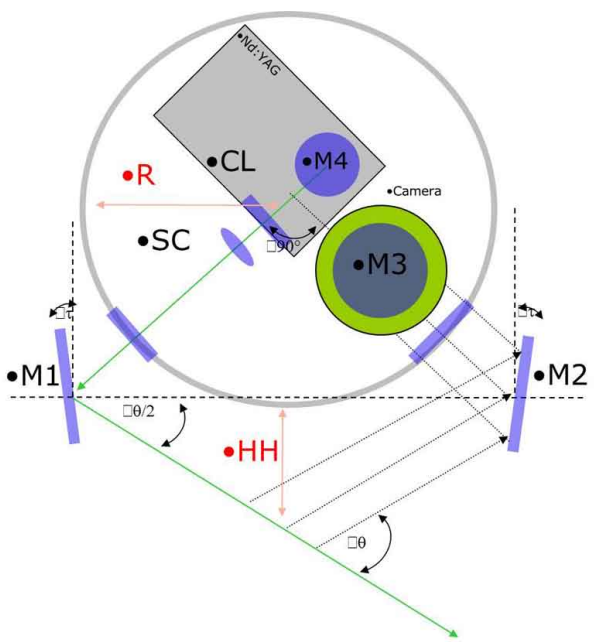

b)

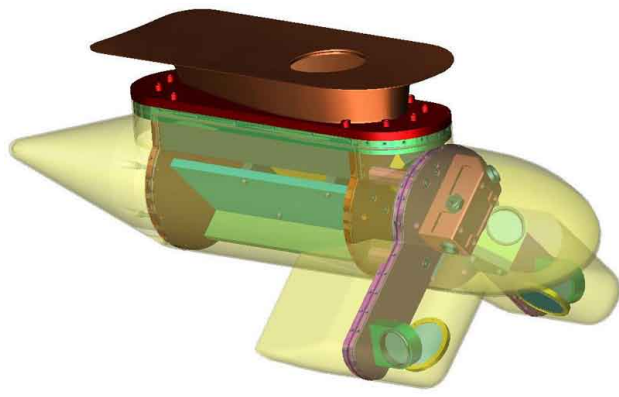

FIG. 2 (a) Front view of the Airborne interferometric Laser Imaging for Droplet Sizing probe (CL, SC: cylindrical, spherical lenses) and (b) 3D design of the probe [17]. The laser and the CCD sensor are in the main central part of the probe. The "two earsextensions" include mirrors and windows. They allow to respect a scattering angle of $\theta=66^{\circ}$, and a distance between the instrument and the measurement volume larger than $10 \mathrm{~cm}$.

\section{ALIDS PROBE QUALIFICATIONS DURING LABORATORY TESTS}

First tests performed at the reception of the ALIDS probe were qualification tests, in order to check that interferograms on each image are morphologically conform to those classically obtained in laboratory. In order to validate the droplet size deduced from the ALIDS probe, out-of-focus interferograms are acquired on a monodispersed drop-by-drop jet (TSI-MDG100). This generator produces drops of uniform size. It works on the same principle as an ink jet printer except that the jet flows continuously. The jet is formed by forcing the liquid through an orifice ( $50 \mu \mathrm{m}$ for present qualification). Liquid jets are naturally unstable and breakup to produce drops with a range of sizes. However, if a disturbance in the form of a square wave is applied to the reservoir supplying the jet at the appropriate frequency, the jet becomes a jet of monodispersed drops. First step of this qualification thus consists in determining the operating point of the generator. Each operating point is a combination of water supply flow rate and frequency applied to the reservoir for which the produced droplets are monodispersed. This is performed with a classical shadow setup. Once the operating points of the MDG 100 generator are precisely determined and characterized by shadowgraphy, this generator is used to validate the diame-

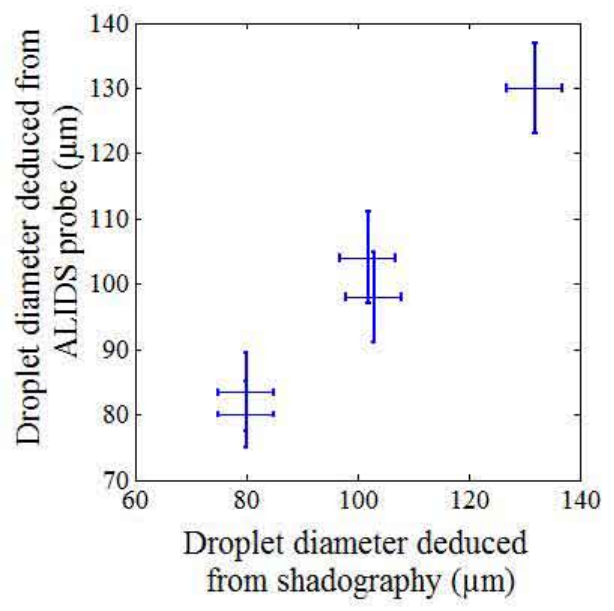

FIG. 3 Comparison between shadow and ALIDS probe measurements.

ter measured by the probe. The ALIDS probe is fixed on an optical table with the so called "ears-extensions" oriented toward the ground. The generator is disposed perpendicularly to the laser sheet so that only one drop intercepts the laser sheet at any time because the inter droplet distance is bigger than the laser sheet width. As a consequence, each image recorded by the probe only contains one interferogram. Interferograms are analysed with a Matlab program [16]. After a Fast Fourier Transform, the frequency of the droplet interferogram is measured. The droplet diameter is deduced using the standard relation for ILIDS analysis (Eq. (2)). Uncertainty on droplet size measurement is mainly due to frequency resolution of the Fast Fourier Transform. The entire procedure is repeated for four operating points of the drop generator. The comparison of diameters obtained using shadowgraphy and ALIDS probe is presented in Figure 3. It shows a good quantitative agreement between both measurements ( $5 \%$ difference between shadowgraphy and the ALIDS probe).

\section{ALIDS PROBE AIRCRAFT INTEGRATION AND FLIGHT TESTS}

The ALIDS probe was implemented on the ATR-42 aircraft of SAFIRE (Service des Avions Francais Instrumentes pour la Recherche en Environnement) [18] in September 2013. Firstly, function tests were performed on the ground in order to check the adjustment of the probe after its integration on the ATR-42 (see the probe on Figure 4). The acquisition system using the aircraft computer was also tested.

An ALIDS test flight was performed during which some examples of out-of-focus images of droplets were obtained (Figure 5). The weather conditions during the flight did not make it possible to fly for a long time inside clouds, as they were below the plane's authorized altitude. Despite this, the images acquired demonstrate that the instrument is well adapted to airborne constraints. The optical adjustments between laser and camera are unaffected by vibrations. The quality of the images is sufficiently high to allow processing even if one can notice that the image contrast is not similar between the two out-of-focus images presented in Figure 5(a) and (b). Indeed, 


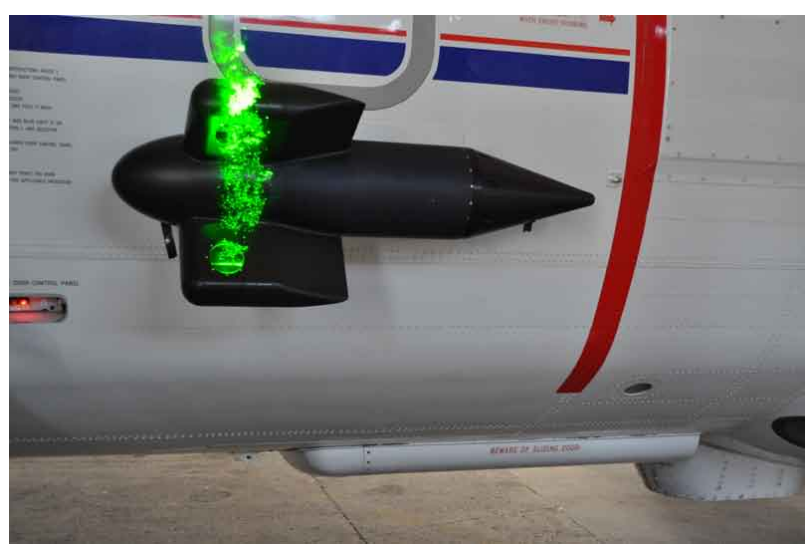

FIG. 4 ALIDS tests on the ground after integration on the ATR- 42 .

in the image of Figure 5(a), it appears that sun light scattering on the cloud induces an important decrease of the image contrast due to the fact that no filter was inserted before the CCD sensor.

The dedicated algorithm was developed and validated in order to process the ILIDS images in real time during airborne tests. A 1D-FFT is performed twice to each ILIDS image: first in the direction perpendicular to the interferometric fringes, and then in the orthogonal direction. The difference of these two 1D-FFT removes any contribution of the image background, and delivers the fringe frequency spectrum [16]. This algorithm is based on the analysis of the global image. It is applied on both images of Figure 5 and the following graph of Figure 6 presents the fringe frequency spectrum deduced from interferogram of Figure 5(a). We deduce a droplet size of $63 \mu \mathrm{m}$ (with the help of Eq. (2)).

Identical processing of the left image of Figure 5 gives a droplet size of $66 \mu \mathrm{m}$.

After this first flight, some technical improvements must be made: an optical filter must be added to remove the sun light scattering on the clouds. Thus, Mie scattering induced by the interaction between the laser sheet $(532 \mathrm{~nm})$ and the droplets will be less masked by the radiation of the solar spectrum. This improvement will be implemented for the next flight. The feasibility of airborne measurements using a prototype probe based on ILIDS technique has been demonstrated. However, if the flight allowed to obtain ILIDS images which have been processed, it has not been possible to realize detailed quantitative measurements. It was not the primary purpose of this first flight. In order to perform comparative tests between ALIDS instrument and other qualified instruments, a new flight campaign will be planned. These new tests will allow a complete qualification of the ALIDS instrument by comparison with measurements of droplet size distribution using a Cloud Particle Imager (CPI) and a Cloud Droplet Probe (CDP).

\section{CONCLUSION}

The ALIDS project, a Joint Research Activity of EUFAR, has been dedicated to the development of a new airborne spectrometer to measure droplet size in clouds. The targeted a)

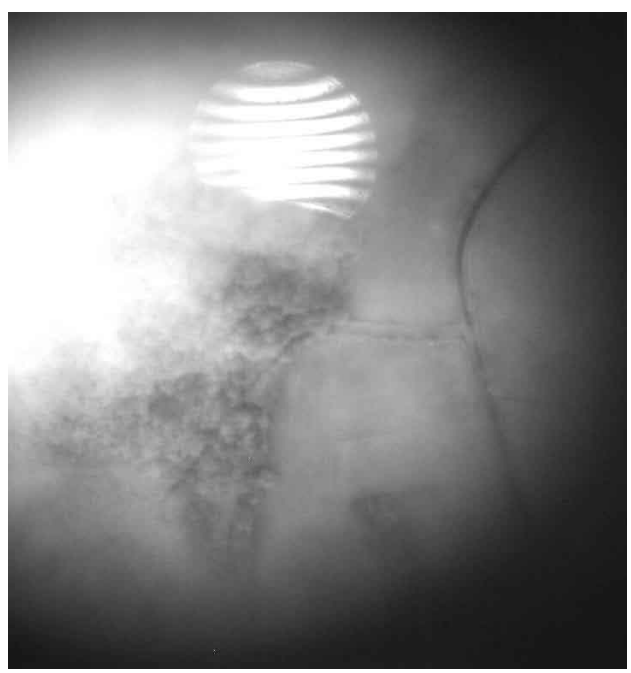

b)

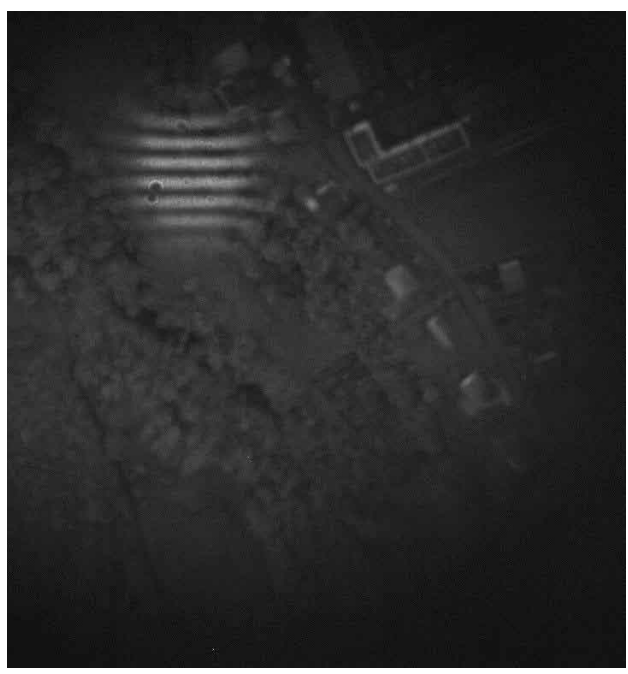

FIG. 5 Examples of ILIDS images obtained in clouds during the flight test.

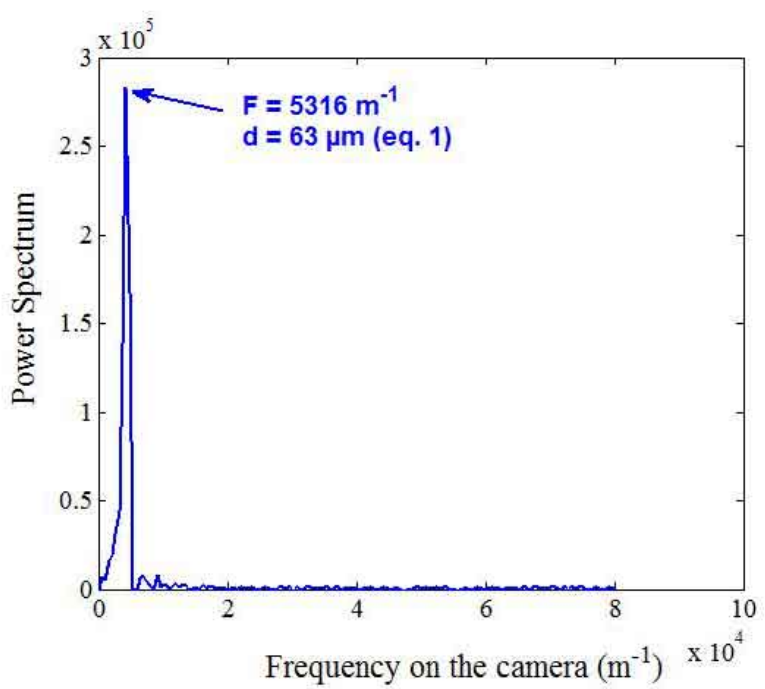

FIG. 6 Spectrum of the image of Figure $5(\mathrm{~b})$.

droplet size range $[20 \mu \mathrm{m}, 200 \mu \mathrm{m}]$ is not yet fully covered by existing airborne instruments. This instrument based on ILIDS technique has been designed, qualified in laboratory 
and tested in airborne conditions. This instrument has a significant evolution potential for further developments. ILIDS principle offers indeed the possibility to change easily the size range of measurements. The continuous improvements in laser and camera technologies will further allow to increase performances of the instrument, such as acquisition rate or spatial resolution of images. New studies are underway within the context of the 7th European Framework Program within High Altitude Ice Crystals (HAIC) project [19] to downsize the ALIDS instrument and to develop a sensor that can characterize icing conditions on aircrafts (for instance super-cooled large droplets and irregular particles, such as ice crystals $[20,21])$.

\section{ACKNOWLEDGEMENTS}

The ALIDS project, Joint Research Activity $N^{\circ} 3$ of EUFAR, was supported by the European Union Seventh Framework Program (FP7, 2007-2013, Grant Agreement $\left.N^{\circ} 227159\right)$. The authors thank the EUFAR project team and the European Commission for supporting this work. Finally, we express our particular gratitude to Jean-Louis Brenguier (Météo-France) for his confidence.

\section{References}

[1] A. Coelho, J. L. Brenguier, and T. Perrin, "Droplet Spectra Measurements with the FSSP-100. Part 1: Coincidence Effects," J. Atmos. Ocean. Tech. 22, 1756 (2005).

[2] J. L. Brenguier, T. Bourrianne, A. Coelho, J. Isbert, R. Peytavi, D. Trevarin, and P. Weschsler, "Improvements of droplet size distribution measurements with the Fast-FSSP," J. Atmos. Ocean. Technol. 15, 1077-1090 (1998).

[3] R. P. Lawson, B. A. Baker, C. G. Schmitt, and T. L. Jensen, “An overview of microphysical properties of Artic clouds observed in May and July during FIRE.ACE," J. Geophys. Res. 106, 14989-15014 (2001).

[4] W. D. Bachalo, and M. J. Houser, "Phase/Doppler spray analyzer for simultaneous measurements of drop size and velocity distribution," Opt. Eng. 23583 (1984).

[5] P. Y. Chuang, E. W. Saw, J. D. Small, R. A. Shaw, C. M. Sipperley, G. A. Payne, and W. D. Bachalo, "Airborne Phase Doppler Interferometry for Cloud Microphysical Measurements," Aerosol Sci. Tech. 42, 685 (2008).

[6] EUFAR, http://www.eufar.net, http://cordis.europa.eu/fp/capacities/home_en.html
[7] G. König, K. Anders, and A. Frohn, "A new light-scattering technique to measure the diameter of periodically generated moving droplets," J. Aerosol Sci. 17, 157-167 (1986).

[8] A. R. Glover, S. M. Skippon, and R. D. Boyle, "Interferometric laser imaging for droplet sizing: a method for droplet-size measurement in sparse spray systems," Appl. Optics 34, 8409-8421 (1995).

[9] T. Kobayashi, T. Kawaguchi, and M. Maeda, "Measurement of spray flow by an improved Interferometric Laser Imaging Droplet Sizing (ILIDS)," in Proceedings of 10th International Symposium Applications of Laser Techniques to Fluid Mechanics, 209-220 (Springer, Lisbon, 2000).

[10] C. Mounaim-Rousselle, and 0. Pajot, "Droplet Sizing by Mie Scattering Interferometry in a Spark Ignition engine," Part. Part. Syst. Char. 16, 160 (1999).

[11] H. C. Van de Hulst, Light Scattering by Small Particles (Dover Publications, Mineola, 1981).

[12] E. Porcheron, P. Lemaitre, A. Nuboer, R. Rochas, and J. Vendel, “Experimental investigation in the TOSQAN facility of heat and mass transfers in a spray for containment application," Nucl. Eng. Des. 237, 1862-1871 (2007).

[13] P. Lemaitre, and E. Porcheron, "Analysis of heat and mass transfers in two-phase flow by coupling optical diagnostic techniques," Exp. Fluids 45, 187-201 (2008).

[14] H. Shen, S. Coetmellec, G. Grehan, and M. Brunel, "ILIDS revisited: elaboration of transfer matrix models for the description of complete systems," Appl. Optics 51, 5357-5368 (2012).

[15] M. Maeda, T. Kawaguchi, and K. Hishida, “Novel interferometric measurement of size and velocity distributions of spherical particles in fluid flows," Meas. Sci. Technol. 11, L13-L18 (2000).

[16] A. Querel, P. Lemaitre, M. Brunel, E. Porcheron, and G. Grehan, "Real-time global interferometric laser imaging for the droplet sizing (ILIDS) algorithm for airborne research," Meas. Sci. Technol.21, 015306 (2010).

[17] COMAT http://www.comat-agora.com/

[18] SAFIRE http://www.safire.fr/

[19] HAIC http://www.haic.eu/

[20] M. Brunel, S. Coetmellec, G. Grehan, and H. Shen, "Interferometric out-of-focus imaging simulator for irregular rough particles," J. Eur. Opt. Soc.-Rapid 9, 14008 (2014).

[21] M. Brunel, S. Conzalez Ruiz, J. Jacquot, and J. van Beeck, "On the morphology of irregular rough particles from the analysis of interferometric out-of-focus images," Opt. Commun. 338, 193-198 (2015). 